PROCEEDINGS OF THE

AMERICAN MATHEMATICAL SOCIETY

Volume 126, Number 2, February 1998, Pages 523-530

S $0002-9939(98) 04164-1$

\title{
SEMICLASSICAL LIMIT OF THE NONLINEAR SCHRÖDINGER EQUATION IN SMALL TIME
}

\author{
E. GRENIER
}

(Communicated by Jeffrey B. Rauch)

\begin{abstract}
We study the semi-classical limit of the nonlinear Schrödinger equation for initial data with Sobolev regularity, before shocks appear in the limit system, and in particular the validity of the WKB method.
\end{abstract}

\section{INTRODUCTION}

We study the behaviour as $h$ goes to zero of solutions $\psi_{h}$ of the nonlinear Schrödinger equation (NLS)

$$
-i h \partial_{t} \psi_{h}-\frac{h^{2}}{2} \Delta_{x} \psi_{h}+f\left(\left|\psi_{h}\right|^{2}\right) \psi_{h}=0
$$

where $x \in \mathbb{R}^{d}$ and $t \in \mathbb{R}^{+}$, with initial data

$$
\psi_{h}(0, x)=a^{0}(x, h) e^{i S^{0}(x) / h},
$$

where $f \in C^{\infty}\left(\mathbb{R}^{+}, \mathbb{R}\right), S^{0}$ is a function of $H^{s}\left(\mathbb{R}^{d}\right)$ (Sobolev spaces) for $s$ large enough, and $a^{0}$ is a function, polynomial in $h$, with coefficients of Sobolev regularity in $x$.

The usual WKB method leads us to look for solutions of the form

$$
\psi_{h}(t, x)=a(t, x, h) e^{i S(t, x) / h}
$$

where

$$
a(t, x, h)=\sum_{j=0}^{+\infty} a_{j}(t, x) h^{j} .
$$

The functions $a_{j}$ satisfy a hierarchy of equations that can be solved in small time (see [1] for more details).

On the other hand [1], with the change of variables

$$
v=\nabla_{x} S+h\left(\bar{a} \nabla_{x} a-a \nabla_{x} \bar{a}\right) / 2 i \rho \quad \text { and } \quad \rho=|a|^{2},
$$

the NLS equation (1.1) is transformed to

$$
\partial_{t} \rho+\nabla_{x} \cdot(\rho v)=0,
$$

Received by the editors August 13, 1996.

1991 Mathematics Subject Classification. Primary 35Q55, 35C20.

Key words and phrases. Nonlinear Schrödinger equations, semiclassical limit.

(C)1998 American Mathematical Society 


$$
\partial_{t} v+\nabla_{x}\left(\frac{|v|^{2}}{2}+f(\rho)\right)=\frac{h^{2}}{2} \nabla_{x}\left(\frac{1}{\sqrt{\rho}} \Delta_{x} \sqrt{\rho}\right),
$$

which is a perturbation of the Euler equations of compressible isentropic fluid mechanics

$$
\begin{gathered}
\partial_{t} \rho+\nabla_{x} \cdot(\rho v)=0, \\
\partial_{t} v+\nabla_{x}\left(\frac{|v|^{2}}{2}+f(\rho)\right)=0 .
\end{gathered}
$$

If $f^{\prime}>0$, this system has smooth solutions on a time interval $\left[0, T^{\star}[\right.$, for initial data with Sobolev regularity [5].

A natural question is then to show the existence of smooth solutions $\psi_{h}$ of (1.1) on a time interval $[0, T]$ independent on $h$, for initial data $a^{0}$ and $S^{0}$ with Sobolev regularity, and to justify the WKB expansion on the same interval of time, $T$ being linked to the existence time of a smooth solution to $(1.5,1.6)$. The case of initial data with analytic regularity has been treated by P. Gérard in [1], and the case $f(\rho)=\rho$ has been investigated in one space dimension for all time by Jin, Levermore and Mc Laughlin in [4], using the integrability of the cubic nonlinear Schrödinger equation.

Notice that with particular assumptions on $d$ and $f$, equation (1.1) has global smooth solutions [2]. This kind of result can however not be used, since the main problem is to obtain bounds uniform in $h$ on the solution $\psi_{h}$, in order to be able to pass to the limit and to justify the WKB hierarchy.

Let us now state the three main theorems.

Theorem 1.1. Let $f \in C^{\infty}\left(\mathbb{R}^{+}, \mathbb{R}\right)$, with $f^{\prime}>0$. Let $s>d / 2+2$, let $S^{0}(x) \in$ $H^{s}\left(\mathbb{R}^{d}\right)$ and $a^{0}(x, h)$ be a sequence of functions uniformly bounded in $H^{s}\left(\mathbb{R}^{d}\right)$. Then there exist $T>0$ and solutions $\psi_{h}(t, x)=a_{h}(t, x) \exp \left(i S_{h}(t, x) / h\right)$ to equation (1.1), with initial data (1.2). Moreover, $a_{h}$ and $S_{h}$ are bounded in $L^{\infty}\left([0, T], H^{s}\right)$ uniformly in $h$.

We can then link $T$ to the existence time of a smooth solution to $(1.5,1.6)$ :

Theorem 1.2. Under the assumptions of Theorem 1.1, if moreover $a^{0}(x, h)$ converges to $a^{0}$ in $H^{s}\left(\mathbb{R}^{d}\right)$ as $h$ goes to 0 , and if system (1.5,1.6) with initial data $\rho(0, x)=\left|a^{0}(x)\right|^{2}$ and $v(0, x)=\nabla_{x} S^{0}(x)$ has a solution in $L^{\infty}\left([0, T], H^{s+2}\left(\mathbb{R}^{d}\right)\right)$, then, for $h$ small enough, there exist solutions to equation (1.1) of the form $\psi_{h}(t, x)$ $=a_{h}(t, x) \exp \left(i S_{h}(t, x) / h\right)$ on $[0, T]$, where $a_{h}$ and $S_{h}$ are bounded in $L^{\infty}\left([0, T], H^{s}\right)$ uniformly in $h$.

We then justify the WKB expansions :

Theorem 1.3. Under the assumptions of Theorem 1.2, if moreover $a^{0}(x, h)$ has an expansion of the form

$$
a^{0}(x, h)=\sum_{j=0}^{N} a_{j}^{0}(x) h^{j}+h^{N} r_{N}(x, h)
$$

where

$$
\lim _{h \rightarrow 0}\left\|r_{N}\right\|_{H^{s}\left(\mathbb{R}^{d}\right)}=0
$$


for $N \in \mathbb{N}$ and $s-2 N-2-d / 2>0$, then, on the time interval $[0, T]$ given by Theorem 1.2,

$$
a_{h}(t, x) \exp \left(i S_{h}(t, x) / h\right)=\sum_{j=0}^{N} a_{j}(t, x) h^{j} \exp (i S(t, x) / h)+h^{N} r_{N}(t, x, h)
$$

as $h$ goes to zero, where $S$ and $a_{j}$ are given by the WKB method, and where

$$
\lim _{h \rightarrow 0}\left\|r_{N}\right\|_{L^{\infty}\left([0, T], H^{s-2 N-2-d / 2}\left(\mathbb{R}^{d}\right)\right)}=0 .
$$

The results of this paper have been announced in [3]. The proofs rely on the following simple idea : instead of looking as usual at solutions $\psi_{h}$ of the form

$$
\psi_{h}(t, x)=a_{h}(t, x) e^{i S(t, x) / h}
$$

where $S$ is independent of $h$, we allow $S$ to depend on $h$, in order to get better equations for $a_{h}$ and $S_{h}$. Namely we will look for solutions $\psi_{h}$ of the form

$$
\psi_{h}(t, x)=a_{h}(t, x) e^{i S_{h}(t, x) / h} .
$$

\section{Proof of Theorem 1.1}

Putting (1.9) in (1.1), we get

$$
\begin{gathered}
-i h \partial_{t} a_{h}+\partial_{t} S_{h} a_{h}-\frac{h^{2}}{2} \Delta_{x} a_{h}-i h \nabla_{x} S_{h} \cdot \nabla_{x} a_{h}-\frac{i h}{2} a_{h} \Delta_{x} S_{h}+\frac{1}{2} a_{h}\left|\nabla_{x} S\right|^{2} \\
+a_{h} f\left(\left|a_{h}\right|^{2}\right)=0,
\end{gathered}
$$

that we split into

$$
\partial_{t} S_{h}+\frac{\left|\nabla_{x} S_{h}\right|^{2}}{2}+f\left(\left|a_{h}\right|^{2}\right)=0
$$

and

$$
\partial_{t} a_{h}-\frac{i h}{2} \Delta_{x} a_{h}+\nabla_{x} S_{h} \cdot \nabla_{x} a_{h}+\frac{1}{2} a_{h} \Delta_{x} S_{h}=0 .
$$

The change of variable $w_{h}=\nabla_{x} S_{h}$ then leads to

$$
\begin{aligned}
& \partial_{t} w_{h}+\left(w_{h} \cdot \nabla_{x}\right) w_{h}+f^{\prime}\left(\left|a_{h}\right|^{2}\right) \nabla_{x}\left|a_{h}\right|^{2}=0, \\
& \partial_{t} a_{h}+\left(w_{h} \cdot \nabla_{x}\right) a_{h}+\frac{1}{2} a_{h} \nabla_{x} \cdot w_{h}=\frac{i h}{2} \Delta_{x} a_{h} .
\end{aligned}
$$

Let $a_{h}^{1}$ be the real part of $a_{h}$ and $a_{h}^{2}$ be its imaginary part. We have

$$
\begin{gathered}
\partial_{t} a_{h}^{1}+\sum_{j=1}^{d} w_{h}^{j} \partial_{j} a_{h}^{1}+\frac{1}{2} a_{h}^{1} \sum_{j=1}^{d} \partial_{j} w_{h}^{j}=-\frac{h}{2} \Delta_{x} a_{h}^{2} \\
\partial_{t} a_{h}^{2}+\sum_{j=1}^{d} w_{h}^{j} \partial_{j} a_{h}^{2}+\frac{1}{2} a_{h}^{2} \sum_{j=1}^{d} \partial_{j} w_{h}^{j}=\frac{h}{2} \Delta_{x} a_{h}^{1}, \\
\partial_{t} w_{h}^{i}+f^{\prime}\left(\left|a_{h}^{1}\right|^{2}+\left|a_{h}^{2}\right|^{2}\right)\left(2 a_{h}^{1} \partial_{i} a_{h}^{1}+2 a_{h}^{2} \partial_{i} a_{h}^{2}\right)+\sum_{i=1}^{d} w_{h}^{j} \partial_{j} w_{h}^{i}=0
\end{gathered}
$$


where $w_{h}^{i}$ is the $i^{t h}$ component of $w_{h}$. This system can be written in the form

$$
\begin{gathered}
\partial_{t} u_{h}+\sum_{i=1}^{d} A^{i}\left(u_{h}\right) \partial_{i} u_{h}=h L\left(u_{h}\right), \\
u_{h}=\left(\begin{array}{c}
a_{h}^{1} \\
a_{h}^{2} \\
w_{h}^{1} \\
\cdots \\
w_{h}^{d}
\end{array}\right), \quad L\left(u_{h}\right)=\left(\begin{array}{c}
-\frac{1}{2} \Delta_{x} a_{h}^{2} \\
\frac{1}{2} \Delta_{x} a_{h}^{1} \\
0 \\
\cdots \\
0
\end{array}\right)
\end{gathered}
$$

and

$$
\begin{gathered}
A\left(u_{h}, \xi\right)=\sum_{j=1}^{d} \xi_{j} A^{j}\left(u_{h}\right) \\
=\left(\begin{array}{ccccc}
\sum_{i=1}^{d} \xi_{i} w_{h}^{i} & 0 & \xi_{1} a_{h}^{1} / 2 & \xi_{2} a_{h}^{1} / 2 & \ldots \\
0 & \sum_{i=1}^{d} \xi_{i} w_{h}^{i} & \xi_{1} a_{h}^{2} / 2 & \xi_{2} a_{h}^{2} / 2 & \ldots \\
2 \xi_{1} a_{h}^{1} f^{\prime} & 2 \xi_{1} a_{h}^{2} f^{\prime} & \sum_{i=1}^{d} \xi_{i} w_{h}^{i} & 0 & \ldots \\
2 \xi_{2} a_{h}^{1} f^{\prime} & 2 \xi_{2} a_{h}^{2} f^{\prime} & 0 & \sum_{i=1}^{d} \xi_{i} w_{h}^{i} & \ldots \\
\ldots & \ldots & \ldots & \ldots & \ddots
\end{array}\right) .
\end{gathered}
$$

The matrix $A\left(u_{h}, \xi\right)$ can be symmetrized by

$$
S=\left(\begin{array}{ccccc}
1 & 0 & 0 & \cdots & 0 \\
0 & 1 & 0 & \cdots & 0 \\
0 & 0 & 1 / 4 f^{\prime} & \cdots & 0 \\
\vdots & \vdots & \vdots & \ddots & \vdots \\
0 & 0 & 0 & \cdots & 1 / 4 f^{\prime}
\end{array}\right)
$$

which is symmetric and positive since $f^{\prime}>0$.

Let $u_{h}=\left(a_{h}^{1}, a_{h}^{2}, w_{h}\right)$ be a solution of system (2.4). We will make classical energy estimates (see for instance [5]), and keep track of the $h$ dependence.

We want to bound $\left(S \partial_{x}^{\alpha} u_{h}, \partial_{x}^{\alpha} u_{h}\right)$ where $\alpha$ is a multi index of length $\leq s$, and $(.,$.$) is the usual L^{2}$ scalar product. We have

$$
\partial_{t}\left(S \partial_{x}^{\alpha} u_{h}, \partial_{x}^{\alpha} u_{h}\right)=\left(\partial_{t} S \partial_{x}^{\alpha} u_{h}, \partial_{x}^{\alpha} u_{h}\right)+2\left(S \partial_{t} \partial_{x}^{\alpha} u_{h}, \partial_{x}^{\alpha} u_{h}\right)
$$

since $S$ is symmetric.

The first term can be bounded by

$$
\left(\partial_{t} S \partial_{x}^{\alpha} u_{h}, \partial_{x}^{\alpha} u_{h}\right) \leq\left|\partial_{t} S\right|_{L^{\infty}}\left\|\partial_{x}^{\alpha} u_{h}\right\|_{L^{2}}^{2},
$$

but

$$
\left|\partial_{t} S\right|_{L^{\infty}} \leq C\left(\left|u_{h}\right|_{L^{\infty}}\right)\left|\partial_{t} u_{h}\right|_{L^{\infty}}
$$

and by Sobolev injections and equation (2.4),

$$
\left|\partial_{t} u_{h}\right|_{L^{\infty}} \leq C\left(\left\|u_{h}\right\|_{s}\right)|| u_{h} \|_{s}
$$

where $s>d / 2+2$ and where

$$
\left\|u_{h}\right\|_{s}^{2}=\sum_{|\alpha| \leq s}\left\|\partial_{x}^{\alpha} u_{h}\right\|_{L^{2}}^{2} .
$$


For the second term we use

$$
\left(S \partial_{t} \partial_{x}^{\alpha} u_{h}, \partial_{x}^{\alpha} u_{h}\right)=h\left(S L\left(\partial_{x}^{\alpha} u_{h}\right), \partial_{x}^{\alpha} u_{h}\right)-\left(S \partial_{x}^{\alpha}\left(\sum_{i=1}^{d} A^{i}\left(u_{h}\right) \partial_{i} u_{h}\right), \partial_{x}^{\alpha} u_{h}\right)
$$

But

$$
\left(S L\left(\partial_{x}^{\alpha} u_{h}\right), \partial_{x}^{\alpha} u_{h}\right)=-\frac{h}{2} \int \partial_{x}^{\alpha} a_{h}^{1} \Delta_{x} \partial_{x}^{\alpha} a_{h}^{2}-\partial_{x}^{\alpha} a_{h}^{2} \Delta_{x} \partial_{x}^{\alpha} a_{h}^{1}=0
$$

by integration by parts.

The second term of (2.5) can be rewritten under the form

$$
\begin{aligned}
\left(S \partial_{x}^{\alpha}\left(\sum_{i=1}^{d} A^{i}\left(u_{h}\right) \partial_{i} u_{h}\right), \partial_{x}^{\alpha} u_{h}\right) & =\left(S \sum_{i=1}^{d} A^{i}\left(u_{h}\right) \partial_{i} \partial_{x}^{\alpha} u_{h}, \partial_{x}^{\alpha} u_{h}\right) \\
& +\left(S\left(\partial_{x}^{\alpha}\left(\sum_{i=1}^{d} A^{i}\left(u_{h}\right) \partial_{i} u_{h}\right)-\sum_{i=1}^{d} A^{i}\left(u_{h}\right) \partial_{i} \partial_{x}^{\alpha} u_{h}\right), \partial_{x}^{\alpha} u_{h}\right) .
\end{aligned}
$$

But

$$
\begin{gathered}
\left(S \sum_{i=1}^{d} A^{i}\left(u_{h}\right) \partial_{i} \partial_{x}^{\alpha} u_{h}, \partial_{x}^{\alpha} u_{h}\right)=-\sum_{i=1}^{d}\left(\partial_{i}\left(S A^{i}\left(u_{h}\right)\right) \partial_{x}^{\alpha} u_{h}, \partial_{x}^{\alpha} u_{h}\right) \\
-\sum_{i=1}^{d}\left(S A^{i}\left(u_{h}\right) \partial_{i} \partial_{x}^{\alpha} u_{h}, \partial_{x}^{\alpha} u_{h}\right)
\end{gathered}
$$

since $S A^{i}\left(u_{h}\right)$ is a symmetric matrix. Therefore

$$
\begin{gathered}
\left|\left(S \sum_{i=1}^{d} A^{i}\left(u_{h}\right) \partial_{i} \partial_{x}^{\alpha} u_{h}, \partial_{x}^{\alpha} u_{h}\right)\right| \leq C\left(\left|u_{h}\right|_{L^{\infty}}\right)|| \partial_{x}^{\alpha} u_{h} \|_{L^{2}}^{2}\left|\nabla_{x} u_{h}\right|_{L^{\infty}} \\
\leq C\left(\left|u_{h}\right|_{L^{\infty}}\right)\left\|\partial_{x}^{\alpha} u_{h}\right\|_{L^{2}}^{2}\left\|u_{h}\right\|_{s}
\end{gathered}
$$

The usual estimates on commutators lead to

$$
\left|\left(S\left(\partial_{x}^{\alpha}\left(\sum_{i=1}^{d} A^{i}\left(u_{h}\right) \partial_{i} u_{h}\right)-\sum_{i=1}^{d} A^{i}\left(u_{h}\right) \partial_{i} \partial_{x}^{\alpha} u_{h}\right), \partial_{x}^{\alpha} u_{h}\right)\right| \leq C\left(\left\|u_{h}\right\|{ }_{s}\right)\left\|u_{h}\right\|_{s}^{2} .
$$

Thus

$$
\partial_{t} \sum_{|\alpha| \leq s}\left(S \partial_{x}^{\alpha} u_{h}, \partial_{x}^{\alpha} u_{h}\right) \leq C\left(\left\|u_{h}\right\|_{s}\right)\left\|u_{h}\right\|_{s}^{2}
$$

for $s>2+d / 2$.

This energy estimate is independent on $h$, and the end of the proof of Theorem 1.1 is straightforward with the help of Gronwall's Lemma. 


\section{Proof of Theorem 1.2}

Assume now that there exists a solution $(\rho, v)$ in $L^{\infty}\left([0, T], H^{s+2}\left(\mathbb{R}^{d}\right)\right)$ to equations $(1.5,1.6)$, on a time interval $[0, T]$, with $s>d / 2+2$, for the initial data

$$
\rho=\left|\lim _{h \rightarrow 0} a_{h}^{0}\right|^{2} \quad \text { and } \quad v=\nabla_{x} S^{0} .
$$

We want to show that there exists a solution to equation (2.4) on the same time interval, for $h$ small enough, a solution which is uniformly bounded in $L^{\infty}\left([0, T], H^{s}\right)$. The limit system of (2.4) is

$$
\partial_{t} u+\sum_{i=1}^{d} A^{i}(u) \partial_{i} u=0
$$

where $u=\left(a_{1}, a_{2}, w\right)$, and admits a solution on a maximal time interval $\left[0, T^{\prime}[\right.$. Let us prove that $T^{\prime}>T$. Assume $T^{\prime} \leq T$ and let $\rho=\left|a_{1}\right|^{2}+\left|a_{2}\right|^{2}$ and $v=w$. As $(\rho, v)$ satisfy $(1.5,1.6)$ with initial data $(3.1)$, we know that $\rho$ and $v$ are in $L^{\infty}\left(\left[0, T^{\prime}\right], H^{s}\left(\mathbb{R}^{d}\right)\right)$; therefore $w \in L^{\infty}\left(\left[0, T^{\prime}\right], H^{s}\left(\mathbb{R}^{d}\right)\right)$. Using $(2.1,2.2)$, we get that $a_{1}$ and $a_{2}$ are in $L^{\infty}\left(\left[0, T^{\prime}\right], H^{s-1}\left(\mathbb{R}^{d}\right)\right)$, which is impossible since $T^{\prime}$ is assumed to be the maximal time of existence. Therefore $T^{\prime}>T$, and system (3.2) has a smooth solution on the time interval $[0, T]$.

Setting $v_{h}=u_{h}-u$, we get

$$
\partial_{t} v_{h}+\sum_{i=1}^{d} A^{i}\left(u+v_{h}\right) \partial_{i} v_{h}+\sum_{i=1}^{d}\left(A^{i}\left(u+v_{h}\right)-A^{i}(u)\right) \partial_{i} u=h L\left(v_{h}\right)+h L(u) .
$$

The matrix $\sum_{i=1}^{d} A^{i}\left(u+v_{h}\right) \xi_{i}$ is symmetrisable and we can make again the energy estimates of the previous section. The term

$$
\sum_{i=1}^{d} A^{i}\left(u+v_{h}\right) \partial_{i} v_{h}
$$

can be treated as above;

$$
\left(S \partial_{x}^{\alpha}\left(\sum_{i=1}^{d}\left(A^{i}\left(u+v_{h}\right)-A^{i}(u)\right) \partial_{i} u\right), \partial_{x}^{\alpha} v_{h}\right)
$$

is bounded by

$$
C\left(\|u\|_{s},\left\|v_{h}\right\|_{s}\right)\left\|v_{h}\right\|_{s}^{2}
$$

Moreover

$$
\left(h S L\left(\partial_{x}^{\alpha} v_{h}\right)+h S L\left(\partial_{x}^{\alpha} u\right), \partial_{x}^{\alpha} v_{h}\right)=\left(h L\left(\partial_{x}^{\alpha} u\right), \partial_{x}^{\alpha} v_{h}\right)
$$

is bounded by

$$
h\|u\|_{\alpha+2}\left\|v_{h}\right\|_{\alpha}
$$

Thus

$$
\partial_{t} \sum_{|\alpha| \leq s}\left(S \partial_{x}^{\alpha} v_{h}, \partial_{x}^{\alpha} v_{h}\right) \leq C\left(\left\|v_{h}\right\|_{s},\|u\|_{s+2}\right)^{2}\left\|v_{h}\right\|_{s}^{2}+h\|u\|_{s+2}\left\|v_{h}\right\|_{s},
$$


for $s>d / 2+2$. Moreover $\left\|v_{h}(t=0)\right\|_{s}$ goes to zero as $h$ goes to 0 ; therefore Gronwall's Lemma shows that for $h$ small enough, there exists a constant $C(h)$ such that

$$
\left\|v_{h}\right\|_{s} \leq C(h) \quad \text { on } \quad[0, T],
$$

with $C(h) \rightarrow 0$ as $h \rightarrow 0$, which ends the proof of Theorem 1.2 .

\section{Proof of Theorem 1.3}

4.1. Zeroth order. From Theorem 1.2, we know that $a_{h}$ and $w_{h}$ are bounded in $L^{\infty}\left([0, T], H^{s}\left(\mathbb{R}^{d}\right)\right)$; thus $\partial_{t} a_{h}$ and $\partial_{t} w^{h}$ are bounded in $L^{\infty}\left([0, T], H^{s-2}\left(\mathbb{R}^{d}\right)\right)$. Therefore, for a subsequence, $a_{h}$ and $w_{h}$ converge uniformly in $L^{\infty}\left([0, T], H^{s-2}\left(\mathbb{R}^{d}\right)\right)$ to $a_{0}^{\prime}$ and $w_{0}^{\prime}$, solutions of

$$
\begin{gathered}
\partial_{t} w_{0}^{\prime}+\left(w_{0}^{\prime} \cdot \nabla_{x}\right) w_{0}^{\prime}+f^{\prime}\left(\left|a_{0}^{\prime}\right|^{2}\right) \nabla_{x}\left|a_{0}^{\prime}\right|^{2}=0, \\
\partial_{t} a_{0}^{\prime}+w_{0}^{\prime} \cdot \nabla_{x} a_{0}^{\prime}+\frac{1}{2} a_{0}^{\prime} \nabla_{x} \cdot w_{0}^{\prime}=0,
\end{gathered}
$$

with initial data $a_{0}^{\prime}=\lim _{h \rightarrow 0} a^{0}(h)$ and $w_{0}^{\prime}=\nabla S^{0}$.

This system admits an unique solution; therefore in fact all the sequence $\left(a_{h}, w_{h}\right)$ converges.

4.2. First order. As in the previous section, we set $v_{h}=u_{h}-u$, and we show by the same energy estimate that

$$
\left\|v_{h}(t)\right\|_{H^{s-2}} \leq h C\left(\|u\|_{L^{\infty}\left([0, T], H^{s}\right)}\right)
$$

for all $t \leq T$. Let then $\tilde{v}_{h}=v_{h} / h$. We see that $\tilde{v}_{h}$ is bounded in $L^{\infty}\left([0, T], H^{s-2}\right)$, and that $\partial_{t} \tilde{v}_{h}$ is bounded in $L^{\infty}\left([0, T], H^{s-4}\right)$. Thus, for a subsequence, $\tilde{v}_{h}$ converges strongly in $L^{\infty}\left([0, T], H^{s-4}\right)$ to a function $u_{1}^{\prime}$. Taking the limit of the equations for $\tilde{v}_{h}$, we obtain that $u_{1}^{\prime}$ solves the linearized problem

$$
\partial_{t} u_{1}^{\prime}+\sum_{i=1}^{d} A^{i}\left(u_{0}^{\prime}\right) \partial_{i} u_{1}^{\prime}+\sum_{i=1}^{d}\left(\nabla A^{i}\left(u_{0}^{\prime}\right) u_{1}^{\prime}\right) \partial_{i} u_{0}^{\prime}=L\left(u_{0}^{\prime}\right)
$$

with initial data

$$
u_{1}^{\prime}=\lim _{h \rightarrow 0} \frac{u_{h}(0)-u_{0}^{\prime}}{h} .
$$

As the solution of this problem is unique, we in fact get the convergence of the whole sequence $\tilde{v}_{h}$ to $u_{1}^{\prime}$.

4.3. Higher order terms. Assume that we already obtained an asymptotic expansion to the order $N$

$$
u_{h}=\sum_{j=0}^{N} u_{j}^{\prime} h^{j}+o\left(h^{N}\right),
$$

the functions $u_{j}^{\prime}$ being in $L^{\infty}\left([0, T], H^{s-2 j}\left(\mathbb{R}^{d}\right)\right)$. Let

$$
\tilde{u}_{h}=\sum_{j=0}^{N} u_{j}^{\prime} h^{j}
$$


and

$$
v_{h}=u_{h}-\sum_{j=0}^{N} u_{j}^{\prime} h^{j}
$$

We write the equation for $u_{h}$, making a Taylor expansion on $A_{i}$ to get

$$
\begin{aligned}
\partial_{t} v_{h} & +\sum_{i=1}^{d} A^{i}\left(\tilde{u}_{h}+v_{h}\right) \partial_{i} v_{h}-\sum_{i=1}^{d}\left(A^{i}\left(\tilde{u}_{h}\right)-A^{i}\left(\tilde{u}_{h}+v_{h}\right)\right) \partial_{i} \tilde{u}_{h} \\
& =h L\left(v_{h}\right)+h^{N+1} B_{h}^{N},
\end{aligned}
$$

where $B_{h}^{N}$ is a function which depends on $\tilde{u}_{h}$ and is bounded in $L^{\infty}\left([0, T], H^{\sigma}\right)$, with $\sigma=s-2 N-2$, uniformly in $h$ (see below). Moreover, by the assumption on the initial data, $h^{-N-1} v_{h}(0)$ is bounded in $H^{s}$. Making again the energy estimates of the previous section, we obtain that $h^{-N-1} v_{h}$ is bounded in $L^{\infty}\left([0, T], H^{s-2 N-2}\right)$.

We then set $\tilde{v}_{h}=v_{h} / h^{N+1}$, and as above, we get that $\tilde{v}_{h}$ converges to some function $u_{N+1}^{\prime}$ as $h$ goes to 0 . To find the equation for $u_{N+1}^{\prime}$, write the term of order $h^{N+1}$ in

$$
\partial_{t}\left(\tilde{u}_{h}+h^{N+1} w_{h}\right)+\sum_{i=1}^{d} A^{i}\left(\tilde{u}_{h}+h^{N+1} w_{h}\right) \partial_{i}\left(\tilde{u}_{h}+h^{N+1} w_{h}\right)-h L\left(\tilde{u}_{h}\right)
$$

is 0 (which enables us to verify that $B_{h}^{N+1}$ is bounded).

4.4. WKB expansion. We have obtained the formal expansion of $a_{h}$ and of $S_{h}$ to an arbitrarily high order. To get back the usual WKB expansion we have only to write the identity of the two following formal series:

$$
\sum_{j=0}^{+\infty} a_{j}(t, x) h^{j} e^{i S(t, x) / h}=\left(\sum_{k=0}^{+\infty} a_{k}^{\prime} h^{k}\right) e^{i \sum_{k=0}^{+\infty} S_{k}^{\prime} h^{k}} .
$$

For instance, $S=S_{0}^{\prime}, a_{0}=a_{0}^{\prime} e^{i S_{1}^{\prime}}$, and $a_{1}=e^{i S_{1}^{\prime}}\left(a_{1}^{\prime}+i S_{2}^{\prime} a_{0}^{\prime}\right)$.

\section{REFERENCES}

1. P. Gérard, Remarques sur l'analyse semi-classique de l'équation de Schrödinger non linéaire, Séminaire EDP de l'École Polytechnique, Palaiseau, France (1992-93), lecture $n^{o}$ XIII. MR 94i:35157

2. J. Ginibre, G. Velo, On the global Cauchy problem for some nonlinear Schrödinger equation, Ann. Inst. H. Poincaré, Anal. non linéaire 1 (1984), 309-323. MR 87a:35164

3. E. Grenier, Limite semiclassique de l'équation de Schrödinger non linéaire en temps petit, C.R. Acad. Sci. Paris, Série I 320 (1995), 691-694. MR 96a:35191

4. S. Jin, C. D. Levermore, D.W. Mc Laughlin, The behaviour of solutions of the NLS equation in the semiclassical limit, Singular limits of dispersive waves, NATO ASI, Series B : Physics, vol. 320, 1994, pp. 235-256. MR 95k:35191

5. A. Majda, Compressible fluid flow and systems of conservation laws in several space variables, Appl. Math. Sci 53, Springer, 1984. MR 85e:35077

Laboratoire d'Analyse Numérique, CNRS - URA 189, Université Paris 6, 4 place Jussieu, 75252 Paris Cedex 05, France

E-mail address: grenier@ann.jussieu.fr 